Kong. Res. J. 2(2) : 62-71, 2015

ISSN 2349-2694

Kongunadu Arts and Science College, Coimbatore.

\title{
ETHNOMEDICINAL APPROACHES FOR TREATING VARIOUS DISEASE BY IRULA TRIBALS, KONBANUR VILLAGE, ANAIKATTI HILLS,THE WESTERN GHATS, COIMBATORE DISTRICT
}

\author{
Ganesan, C.M. ${ }^{1 *}$, A. Manigandan ${ }^{1}$ and G. Thirumaran ${ }^{2}$ \\ 1Department of Botany, Kongunadu Arts and Science College, Coimbatore - 641029. \\ ${ }^{2}$ Kandasamy Kandar Higher Secondary School, Velur, Namakkal - 638182. \\ *E.mail: bioganesan@gmail.com
}

\begin{abstract}
Ethno-medicine means the medical practices for the treatment of ethnic or aborigine people for their health care needs. Indigenous traditional Knowledge is an integral part of the culture and history of a local community. It is evolved through years of regular experimentation on the day to day life and available resources surrounded by the community. The present paper documented 85 ethno-medicinal plants of Konbanur village, Anaikatti, Coimbatore district, the Western Ghats, Tamil Nadu belonging to 48 families were used by the Irula tribals for various diseases and food. The conventional ethno medicinal plants were mostly used for different inflammation, cough and cold, leucoderma, different skin diseases, ulcers and leprosy. The medicinal plants used by the Irula tribal traditional users of Konbanur village, Anaikatty hills are arranged alphabetically followed by botanical name, family, local name and medicinal uses.
\end{abstract}

Keywords: Ethnomedicine, Irula tribals, Anaikatti hills.

\section{INTRODUCTION}

India is endowed with a variety of natural resources. All along the West coast the Western Ghats are sprawling. The entire Western Ghats is known for its biodiversity, richness and endemism of different species. India harbours about 15\% $(3000-3500)$ out of 20,000 medicinal plants of the world. About $90 \%$ of these are found growing wild in different climatic regions of the country. The tribal and rural populations of India are, to a large extent, dependent on medicinal plants not only to meet their own healthcare needs by self-medication, but also for their livestock.The Western Ghats is richly credited with varied kind of vegetation and unimaginable topographical features. There are about 2,000 plant species that has been found to possess the medicinal value, in all the four systems of indigenous medicine, viz, Ayurveda, Unani, Siddha, and Homeopathy. Irulas are a small tribal community that is part of the Dravidian language group that is spoken in South-Eastern India. They are recognized as a Scheduled Tribe (ST) by the Government of India (Sasi et al., 2011; Ragupathy and Newmaster, 2009). The Irulas are the Dravidian inhabitants and one among the 36 sub-tribal communities in Tamil Nadu that holds the population about 26,000 Irulas living in Tamil Nadu, out of the total population of 558 lakh in the state (Department of Tribal Welfare of Tamil Nadu, Statistic table, July 2006), which is less than $0.5 \%$ of the entire state's population (Census of India, 1991 and 2001). The study area Konbanur village, Anaikatti $\left(11^{\circ} 6^{\prime} \mathrm{N}, \quad 76^{\circ} 45^{\prime} \mathrm{E}\right)$. is occupied 250 acres site constitutes a part of the large two square kilometers catchment area. Two hill slopes, northern and southern, also form a part of NBR park. The hills elevate to a height of 80 to 120 metres from the valleys (Fig. 1).

\section{MATERIALS AND METHODS}

Each and every ethnobotanical work has various activities. They are field trip, observations, identification retrieving the medicinal properties and mode of preparation of drug from the plants by Irula tribal community.

The present work is the outcome of intensive field studies undertaken in hamlet inhabited by Irulas community. Explorative field trips were regularly made once in a month of the study area to all habitants to elicit information on medicinal plant used to treat various ailments. Folklore medico botanical investigations were carried out according to the method adopted by Schultes $(1960,1962) ;$ Jain (1989) and Martin (1995). Fieldwork is the most significant aspect in this type of study. Extensive field trips were conducted to remote rural settlements. From each village, two or three local herbal healers were interviewed to elicit first hand information in respect of the plant/plant product curing various diseases. The voucher specimen plants collected were identified with the help of Flora of Presidency of 
Madras by Gamble (1936) and Flora of Tamilnadu and Carnatic by Mathew (1983).

The medicinal plants collected in this way are tabulated. They are documented, both family and genus are arranged according to the alphabetical order. The botanical names followed by author citation and synonyms of the plant species, local name of the plant species also provided. Most of the plants are used as a medicine rest of them served as an edible plants.

\section{RESULTS AND DISCUSSION}

The present study was carried out in the Konbanur village of Anaikatti hills, the Western Ghats, Coimbatore District. Fieldwork is the most significant aspect in this type of study. Extensive field trips were conducted to remote rural settlements. From each village, two or three local herbal healers were interviewed to elicit first hand information in respect of the plant/plant product curing various diseases. In Table 1, data obtained from the field survey are presented. In this study 85 plant species belonging to 48 families have been recorded. Many plant species belonging to families of Solanaceae, Asteraceae and Amarandhaceae are frequently used (Table 2). The informations collected from this study are in agreement with the previous reports (Pushpangadan and Atal, 1984, Kala, 2005; Jain and Shrivastava, 2001; Ayyanar and Ignacimuthu, 2005; Sandhya et al., 2006; Ignacimuthu et al., 2006). For common ailments such as fevers, stomach ache and respiratory disorders, skin diseases, joint pains, hair loss, dysentery, diarrhea, snakebite, jaundice and malaria more number of medication were used. On the other hand, few were used to complicated problems such as heart diseases, kidney disorders skin diseases, cancer and diabetes. The knowledge informants were taken to the field and information on medicinal plants was recorded. The informants were asked to explain therapies of the diseases and to list plants they employ (Table 3-5). In this investigation, there are 20species belonging to 17 families and 18 Genera were reported by the local practice for the treatment of common heart diseases (Table 3).Among them, 17families represents atleast single species each. Nearly 20 species, they are using for the treatment of common stomach problems which belonging into 12 families and 20 Genera (Table 4) and The Irula communities of the study area selectively used around 15 families with their 19 plant species especially for the treatment of kidney disorders which is belonging into (Table 5) Amarandhaceae, Asclepiadaceae, Cucurbitaceae,
Lamiaceae, Fabaceae, Malvaceae, Menispermaceae and Nyctaginaceae etc.,

For each plant species complete documentation of folklore medicinal information including medicinal property, their vernacular names, family, parts of used, uses and their identified phytochemical compounds was recorded (Martin, 1995).

The most important aspect of the Irula tribal medicine is that fresh plant material is used for the preparation of medicine. Alternatively, if the fresh plant parts are not available, dried plant materials are used. For this reason several plants served as edible food and alternative remedy to cure a more than single diseases. From this study it is clear that Irula tribal possess innate ability to discern the character of plants and exploit the plant resources to meet their health care needs.

\section{Fig. I. Location of the study area.}

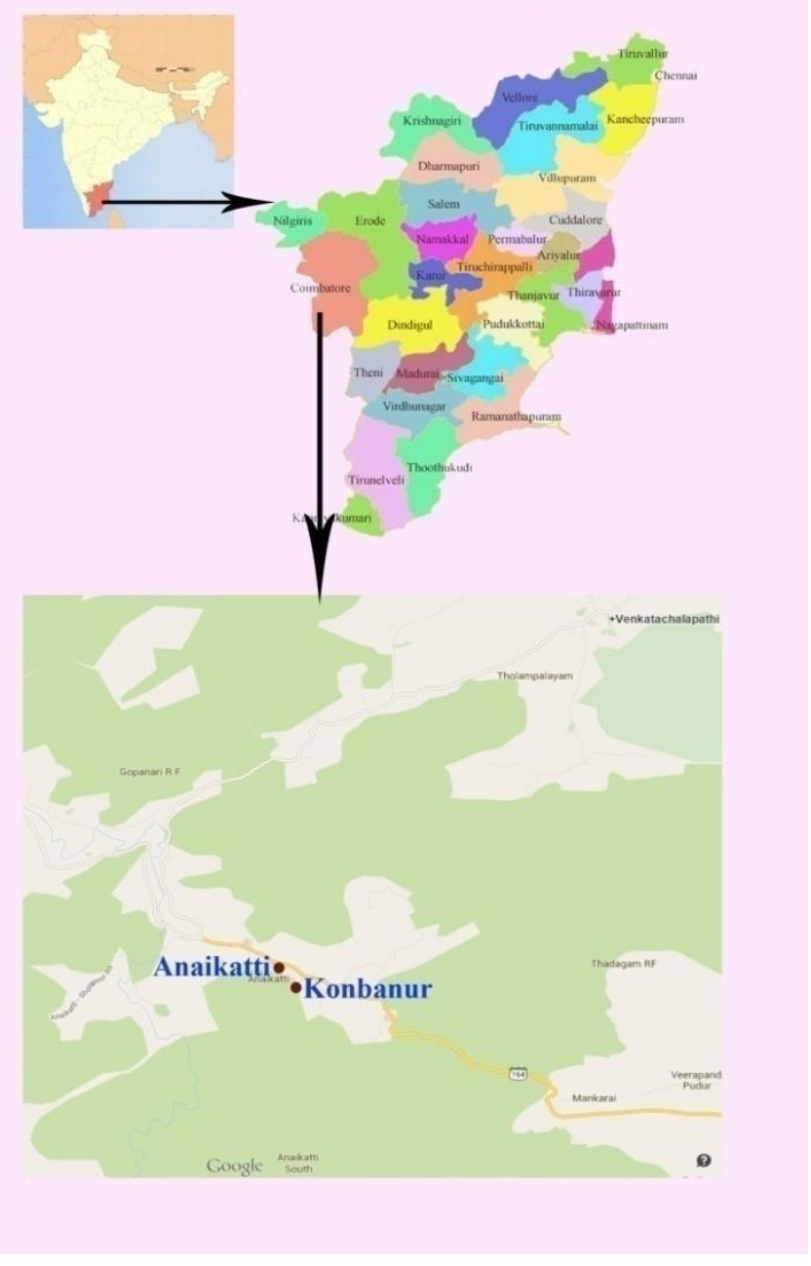


Table 1. List of Ethnomedicinal plants used by Irula tribal in study area.

\begin{tabular}{|c|c|c|c|c|}
\hline S.No & Botanical Name & Family & Vernacular Name & $\begin{array}{l}\text { Parts used ,Mode of Preparation, Ethno } \\
\text { medicinal uses and some other plants used } \\
\text { as ingredients }\end{array}$ \\
\hline 1 & AbelmoschusesculandusL. & Malvaceae & Bhendhi & Protect from asthma and diabetes \\
\hline 2 & AbrusprecatoriusL. & Fabaceae & Rosary pea, Ratti & Used in stomach pains and diarrhea \\
\hline 3 & AbutilonindicumLinn. & Malvaceae & Thuthi & Protect from Piles and Pulmonary tuberculosis \\
\hline 4 & AchyranthesasperaLinn. & Amaranthaceae & Chirchitta & $\begin{array}{l}\text { Useful in treatment of Vomiting, Cough, } \\
\text { Dysentery }\end{array}$ \\
\hline 5 & Aconitum heterophyllumL. & Fabaceae & Athividayam & Extracts used for treating Snakebite, Fever \\
\hline 6 & AcoruscalamusL. & Acoraceae & Vasambu & $\begin{array}{l}\text { Rhizome used for cough \& fever. Leaf used for } \\
\text { Diuretic }\end{array}$ \\
\hline 7 & Adina cordifolia(Roxb.) & Rubiaceae & Kadami & Medicine for Stomach-ache, cold cough, fever \\
\hline 8 & Aeglemarmelos(L.) Correa & Rutaceae & Vilvam & Fruits used for Dysentery \\
\hline 9 & Aervalanata $\mathrm{L}$. & Amarandhaceae & Kanpulai & Leaf juice cure kidneystone \\
\hline 10 & AgeratumconyzoidesL. & Asteraceae & Chick weed & $\begin{array}{l}\text { Treating for stomach pain and antifungal } \\
\text { disease }\end{array}$ \\
\hline 11 & Allium ceba L. & Liliaceae & Onion & $\begin{array}{l}\text { To relieve congestions especially in lungs \& } \\
\text { bronchial tract. }\end{array}$ \\
\hline 12 & Allium sativum L. & Liliaceae & Garlic & To lower blood pressure and cholesterol. \\
\hline 13 & AmarandhuscaudatusL. & Amarandhaceae & Cirukeerai & Avoid diarrhea done by its powder \\
\hline 14 & AmaranthusspinosusL. & Amaranthaceae & Mullu & Leaf juice used for Diuretic \& Digestion \\
\hline 15 & Andrographispaniculata(Burm.f.) & Acanthaceae & Siriyanangai & $\begin{array}{l}\text { Leaf paste mixed with milk internally taken for } \\
\text { snake bite }\end{array}$ \\
\hline 16 & Aristolochia bracteolate Lam. & Aristolochiaceae & Aaduthinnapaalai & $\begin{array}{l}\text { Leaf Paste used externally on the wound of } \\
\text { snake bite }\end{array}$ \\
\hline 17 & ArtocarpusheterophyllusLam. & Moraceae & Palamaram & Leaf juice used for taken internally for ulcer \\
\hline 18 & Basella alba L. & Basellaceae & Kodipasalai & $\begin{array}{l}\text { Leaves boiled in water and taken internally to } \\
\text { cure piles }\end{array}$ \\
\hline 19 & BoerhaaviadiffusaL. & Nyctaginaceae & Mukkurttaikkoti & Taken for treatment of abdominal pain,tumors \\
\hline 20 & Bryophyllumpinnatum (Lam.)oken & Crassulaceae & Malaikali & Cure kidney stone and Cough \\
\hline 21 & Burberis vulgaris Linn. & Berberidaceae & Jaundice barberry & Fruit used to reinforce the heart and liver \\
\hline 22 & CaesalpiniapulcherrimaLinn. & Fabaceae & Peacock Flower & $\begin{array}{l}\text { Focusing the diseases like asthma, malaria, } \\
\text { kidney stone }\end{array}$ \\
\hline 23 & Caeselpiniabonduc (L.) Roxb. & Caesalpinaceae & Kazhichikai & $\begin{array}{l}\text { Seed used for Fever. Leaf juice used for } \\
\text { diabetics }\end{array}$ \\
\hline 24 & Camellia sinensis (L.) Kuntze & Berberidaceae & Tea plant & $\begin{array}{l}\text { Tea used for cancer, heart disease, liver } \\
\text { disease }\end{array}$ \\
\hline 25 & Canna indicaL. & Scitaminaceae & Kalvazhai & Root juice are used for diuretic \& digestion \\
\hline
\end{tabular}




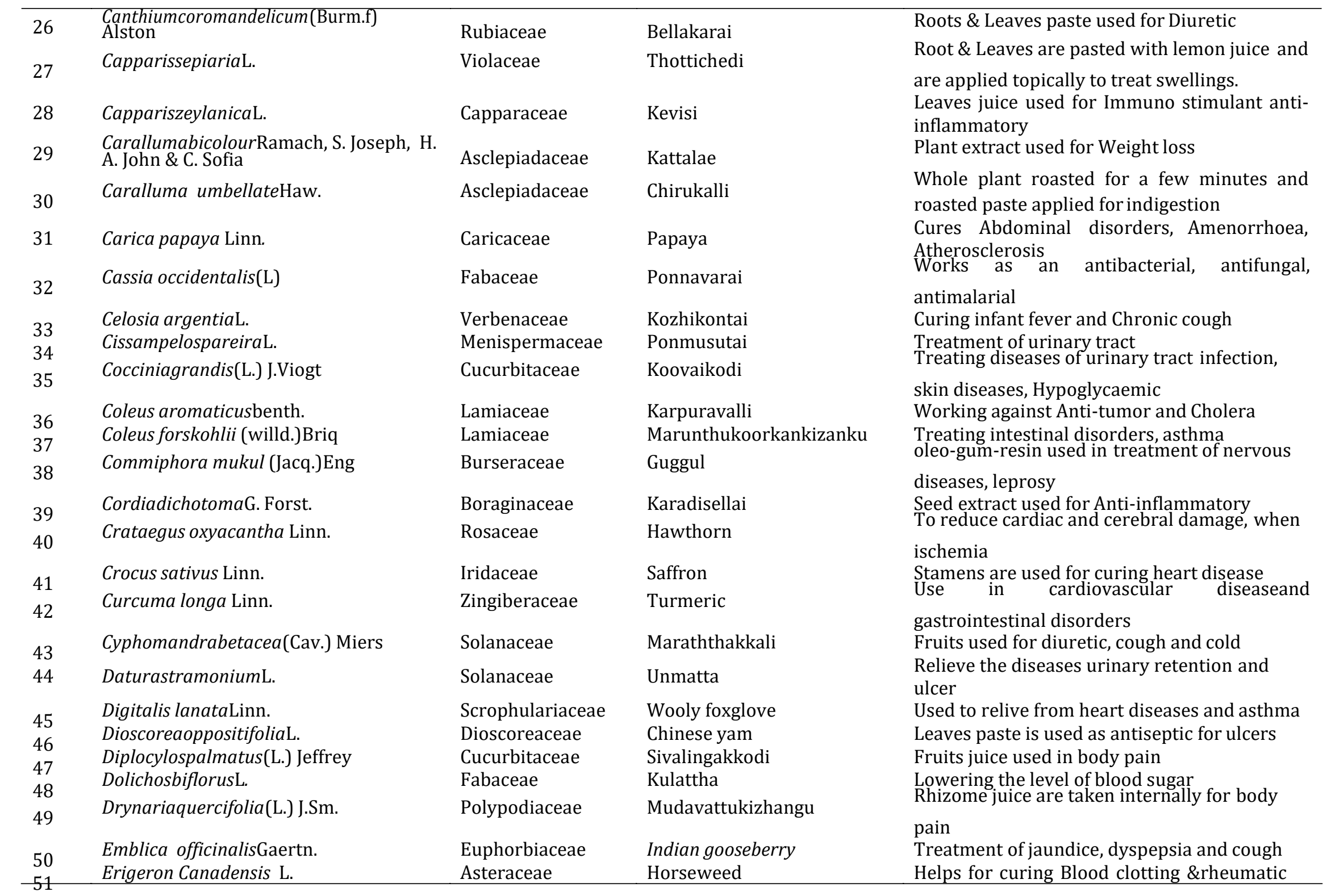




\begin{tabular}{|c|c|c|c|c|}
\hline 52 & GloriosasuperbaL. & Liliaceae & Kanvalipoo & $\begin{array}{l}\text { complaints } \\
\text { Rhizome paste is applied treat wounds. }\end{array}$ \\
\hline 53 & Glycosmispentaphylla(Retz.) Dc. & Rutaceae & Melaekulukki & $\begin{array}{l}\text { Used for cough, rheumatism, anemia and } \\
\text { jaundice. }\end{array}$ \\
\hline 54 & Gompherna serrate L. & Amarandhaceae & Arasan con todo & Cures the Kidney problems and live disorders \\
\hline 55 & Guizotiaabyssinica(L.f.) Cass. & Asteraceae & Malaiellu & Treatment for Stomach ache \\
\hline 56 & Hemidesmusindicus $\mathrm{L}$. & Asclepiadaceae & Nanari & $\begin{array}{l}\text { Refrigerant and for kidney and urinary } \\
\text { disorders }\end{array}$ \\
\hline 57 & Inula racemosa $\mathrm{HOOK.} \mathrm{F}$ & Asteraceae & Sunspear & Roots are powerful biological activity. \\
\hline 58 & JatrophamultifidaL. & Euphorbiaceae & Churakkalli & Protects fromStomach ache, burn \\
\hline 59 & JusticiaadhatodaL. & Acanthaceae & Aadhatodai & $\begin{array}{l}\text { Leaf juice from this plant used for cough, fever } \\
\text { and diarrhea }\end{array}$ \\
\hline 60 & KalanchoepinnataL. & Crassulaceae & Ranakalli & Medicine for curing kidney diseases \\
\hline 61 & Lagenariasiceraria L. & Cucurbitaceae & Surakkai & $\begin{array}{l}\text { Treating diseases like Diabetic,Doarrhea and } \\
\text { digestive problem }\end{array}$ \\
\hline 62 & Madhucalongifolia(Koenig) & Sapotaceae & Iluppai & Medicine for diabetes, Painkiller, Skin diseases \\
\hline 63 & MatricarrecutitaL. & Asteraceae & Chamomile & $\begin{array}{l}\text { Cures the digestive problems and acts as an } \\
\text { anti-inflammatory, anti-spasmodic. }\end{array}$ \\
\hline 64 & MomordicacharantiaL. & Cucurbitaceae & Pakkrkai & Cure kidney stone. \\
\hline 65 & Moringa oleifera $\mathrm{L}$. & Moringaceae & Murungai & Stabilize blood pressure and make strengthen \\
\hline 66 & Nelumbo nucifera Gaertn & Nymphaeaceae & Indian Lotus & $\begin{array}{l}\text { Treatment of diarrhea, tissue inflammation } \\
\text { and haemostasis }\end{array}$ \\
\hline 67 & Pachygoneovata (Poir.) Diels & Menispermaceae & Perungkaattukodi & Seeds powder used for Snake bites \\
\hline 68 & Pergulariadaemia(Forsk) Chiv & Asclepiadaceae & Veliparuthi & $\begin{array}{l}\text { Treating the diseases like malarial intermittent } \\
\text { fevers, toothaches }\end{array}$ \\
\hline 69 & Phyllandhusniruri L. & Phyllandhaceae & Keezhanelli & Brain tumor andJaundice \\
\hline 70 & Piper longum L. & Piperaceae & Long pepper & $\begin{array}{l}\text { Therapeutic agent for Alzheimer disease, Anti- } \\
\text { stress }\end{array}$ \\
\hline 71 & Psidium guajava L. & Myrtaceae & Guava & Rich in antioxidant properties \\
\hline 72 & Punica granatum L. & Puniacaceae & Pomegranate, & $\begin{array}{l}\text { Focusing on treatment of diabetics and } \\
\text { prevention of cancer, cardiovascular disease }\end{array}$ \\
\hline 73 & RicinuscommunisL. & Euphorbiaceae & Castor & Protect liver damage from certain poisons \\
\hline 74 & RiveahypocrateriformisChoisy & Convolulaceae & Mustae & Leaves paste used for diarrhea \\
\hline 75 & Scillahyacinthina(Roth) Macbr. & Liliaceae & Kattuvengayam & $\begin{array}{l}\text { Paste made from bulb applied externally for } \\
\text { body pain }\end{array}$ \\
\hline 76 & Scopariadulcis L. & Scrophuraliaceae & Sarkaraivempu & Cure kidney stone. \\
\hline 77 & SolanumnigrumL. & Solanaceae & Makoi & $\begin{array}{l}\text { Having antiulcer properties cures stomach } \\
\text { diseases }\end{array}$ \\
\hline 78 & SolanumrudepannumDunal & Solanaceae & Toothuvalai & Leaf juice is taken orally for cough and fever \\
\hline
\end{tabular}




\begin{tabular}{|c|c|c|c|c|}
\hline 79 & StrychnospotatorumL.f. & Loganiaceae & Sillakottai & The whole plants used for Urinary \& Kidney \\
\hline 80 & TerminaliaarjunaW. \& A. & Combretaceae & White Marudah & Protects the heart, strengthens circulation \\
\hline 81 & TerminaliachebulaRetz. & Combretaceae & Haritaki & Works as anAntioxidant,Antibacterial, \\
\hline 82 & Tribulusterrestis L. & Zygophyllaceae & Nerunji & Protects the liver and kidney \\
\hline 83 & WithaniasomniferaDunal & Solanaceae & Winter cherry & Increases hemoglobin content in the blood \\
\hline 84 & ZingiberofficinaleRoscoe. & Zingiberaceae & Ginger & Useful in fighting heart disease, cancer \\
\hline 85 & Zizphus jujube (L.) & Rhamnaceae & Ber & $\begin{array}{l}\text { Increase physical stamina and cures the liver } \\
\text { disorders }\end{array}$ \\
\hline
\end{tabular}

Table 2. List of families with number of species in study area.

\begin{tabular}{|c|c|c|c|c|c|}
\hline S.No & Name of the Family & $\begin{array}{c}\text { No. of species } \\
\text { present in each family }\end{array}$ & S.No & Name of the Family & $\begin{array}{c}\text { No. of species } \\
\text { present in each family }\end{array}$ \\
\hline 1 & Acanthaceae & 2 & 25 & Malvaceae & 2 \\
\hline 2 & Acoraceae & 1 & 26 & Menispermaceae & 2 \\
\hline 3 & Amarandhaceae & 5 & 27 & Moraceae & 1 \\
\hline 4 & Aristolochiaceae & 1 & 28 & Moringaceae & 1 \\
\hline 5 & Asclepiadaceae & 4 & 29 & Myrtaceae & 1 \\
\hline 6 & Asteraceae & 5 & 30 & Nyctaginaceae & 1 \\
\hline 7 & Basellaceae & 1 & 31 & Nymphaeaceae & 1 \\
\hline 8 & Berberidaceae & 1 & 32 & Phyllandhaceae & 1 \\
\hline 9 & Boraginaceae & 1 & 33 & Piperaceae & 1 \\
\hline 10 & Burseraceae & 1 & 34 & Polypodiaceae & 1 \\
\hline 11 & Caesalpinaceae & 1 & 35 & Puniacaceae & 1 \\
\hline 12 & Capparaceae & 1 & 36 & Rhamnaceae & 4 \\
\hline 13 & Caricaceae & 1 & 37 & Rosaceae & 1 \\
\hline 14 & Combretaceae & 2 & 38 & Rubiaceae & 2 \\
\hline 15 & Convolulaceae & 1 & 39 & Rutaceae & 2 \\
\hline 16 & Crassulaceae & 2 & 40 & Sapotaceae & 1 \\
\hline 17 & Cucurbitaceae & 4 & 41 & Scitaminaceae & 1 \\
\hline 18 & Dioscoreaceae & 1 & 42 & Scrophulariaceae & 2 \\
\hline 19 & Euphorbiaceae & 3 & 43 & Solanaceae & 5 \\
\hline 20 & Fabaceae & 2 & 44 & Theaceae & 1 \\
\hline 21 & Iridaceae & 1 & 45 & Verbenaceae & 1 \\
\hline 22 & Lamiaceae & 2 & 46 & Violaceae & 1 \\
\hline 23 & Liliaceae & 4 & 47 & Zingiberaceae & 2 \\
\hline 24 & Loganiaceae & 1 & 48 & Zygophyllaceae & 1 \\
\hline
\end{tabular}


Table 3. List of medicinal plants used by Irula tribal for the treatment of heart diseases.

\begin{tabular}{|c|c|c|c|c|c|}
\hline S.No & Botanical Name & Common name & $\begin{array}{l}\text { Name of the } \\
\text { Family }\end{array}$ & Parts used & Chemical Constituents \\
\hline 1 & Allium ceba $\mathrm{L}$. & Onion & Liliaceae & $\begin{array}{l}\text { Bulb \& } \\
\text { Leaves }\end{array}$ & $\begin{array}{l}\text { Sulphur compounds(Ajoene, allyl sulfides, and } \\
\text { vinyldithiins), quercetin\& Allicin(diallyl } \\
\text { disulphide oxide) }\end{array}$ \\
\hline 2 & Allium sativum $\mathrm{L}$. & Garlic & Liliaceae & Bulb & $\begin{array}{l}\text { Sulphur compounds,(Ajoene, allyl sulfides, and } \\
\text { vinyldithiins)\&Allicin }\end{array}$ \\
\hline 3 & Burberis vulgarisLinn. & Jaundice barberry & Berberidaceae & Bark \& Root & Berberine \\
\hline 4 & $\begin{array}{lll}\text { Camellia } & \text { sinensis } & \text { (L.) } \\
\text { Kuntze } & & \end{array}$ & Tea plant & Theaceae & $\begin{array}{l}\text { Leaves } \\
\& \\
\text { Leaf buds }\end{array}$ & $\begin{array}{l}\text { Epicatechin (EC),Epigallocatechin } \\
\text { Epicatechin-3-gallate (ECG), and } \\
\text { Epigallocatechin-3-gallate (EGCG) }\end{array}$ \\
\hline 5 & $\begin{array}{l}\text { Coleus } \\
\text { forskohlii(willd.)Briq }\end{array}$ & $\begin{array}{l}\text { Marunthu } \\
\text { koorkankizanku }\end{array}$ & Lamiaceae & $\begin{array}{l}\text { Tuberous } \\
\text { root }\end{array}$ & Forskohlin,Arjunic acid \\
\hline 6 & $\begin{array}{l}\text { Commiphora } \\
\text { mukul((jacq.)Eng. } \\
\text { Crataeaus }\end{array}$ & Guggul & Burseraceae & $\begin{array}{l}\text { Gum } \\
\text { \&Resin }\end{array}$ & Guggulsterones,Z-guggulsterone, Guggulipids \\
\hline$\gamma$ & oxyacanthaLinn. & Hawthorn & Rosaceae & $\begin{array}{l}\text { Berries, Leaves } \\
\text { \&Flowers }\end{array}$ & $\begin{array}{l}\text { Oligomeric proanthocyanidins, } \\
\text { Catechin, Quercetin,Epicatechin }\end{array}$ \\
\hline 8 & Crocus sativus Linn. & Saffron & Iridaceae & Stigmas & Crocetin, Picrocrocin \\
\hline 9 & Curcuma longa Linn. & Turmeric & Zingiberaceae & Rhizome & Curcumin(diferuloylmethane) C3 \\
\hline 10 & Digitalis lanataLinn. & Grecin foxglove & Scrophulariaceae & Leaves & Cardiac glycosides \\
\hline 11 & Emblica officinalisGaertn. & Amalaki, amla & Euphorbiaceae & Fruit & Vitamin C, Gallic acid, Emblicanin A,B \\
\hline 12 & Inularacemosa HOOK. F & Indian elecampane & Asteraceae & $\begin{array}{l}\text { Root\& } \\
\text { Rhizome }\end{array}$ & Alantolactone, isoalantolactone \\
\hline 13 & Nelumbo nucifera Gaertn & $\begin{array}{l}\text { Indian } \\
\text { Lotus }\end{array}$ & Nymphaeaceae & $\begin{array}{l}\text { Flowers } \\
\text { \&Rhizome }\end{array}$ & Quercetin,Luteolin \\
\hline 14 & Piper longum L. & $\begin{array}{l}\text { Long } \\
\text { pepper,Thippali }\end{array}$ & Piperaceae & $\begin{array}{l}\text { Fruit\& } \\
\text { Root }\end{array}$ & Piperlongumine \\
\hline 15 & Psidium guajava L. & Guava & Myrtaceae & $\begin{array}{l}\text { Fruit } \\
\text { \&Leaves }\end{array}$ & $\begin{array}{l}\text { Quercetin, } \\
\text { Lycopene,vitamin C }\end{array}$ \\
\hline 16 & Punica granatum $\mathrm{L}$. & Pomegranate & Puniacaceae & Fruits\& flowers & $\begin{array}{l}\text { Hexahydroxydiphenic acid,Gallic } \quad \text { acid, } \\
\text { guercețin, Punicic accid. }\end{array}$ \\
\hline 17 & TerminaliaarjunaW. \& A. & Maruthamaram & Combretaceae & Bark & Arjunolic acid,Arjunic àcid, Glycosides, \\
\hline 18 & TerminaliachebulaRetz. & Haritaki & Combretaceae & $\begin{array}{l}\text { Fruit, Bark } \\
\text { \&seed }\end{array}$ & $\begin{array}{l}\text { Gallic acid, oligomeric proanthocyanidins } \\
\text { Pentacyclictriterpenes, vasicine } \\
\text { vasicinone,Ellagic acid,chebulic acid }\end{array}$ \\
\hline 19 & WithaniasomniferaDunal & $\begin{array}{l}\text { Winter cherry, } \\
\text { Ashwagandha }\end{array}$ & Solanaceae & $\begin{array}{l}\text { Tuber } \\
\text { \&Root }\end{array}$ & Withaferin A \\
\hline 20 & ZingiberofficinaleRoscoe. & Ginger & Zingiberaceae & Root & Galanolactone \\
\hline
\end{tabular}


Table 4. List of medicinal plants used by Irula tribal for the treatment of stomach disorders.

\begin{tabular}{|c|c|c|c|c|c|}
\hline S.No. & Botanical name & Name of the family & Common name & Part used & chemical constitution \\
\hline 1 & AbrusprecatoriusL. & Fabaceae & Kuntrymani & Seed & 2,3-diphospho-d-glyceric Acid \\
\hline 2 & AchyranthesasperaLinn. & Amaranthaceae & Chirchitta & whole plant & C-glycosides \\
\hline 3 & Aconitum heterophyllumL. & Fabaceae & Athividayam & whole plant & Heterophylline,Hetisine \\
\hline 4 & Adina cordifolia(Roxb.) & Rubiaceae & Kadami & leaf, flower & Rhamnopyranosyl \\
\hline 5 & Ageratum conyzoides $\mathrm{L}$ & Asteraceae & Chick weed & whole plant & Leucoanthocyanins \\
\hline 6 & Caesalpiniapulcherrima Linn. & Fabaceae & Peacock Flower & Leaf & Terpinene \\
\hline 7 & Carica papaya Linn & Caricaceae & Papaya & fruit, seed & cardiac glycosides \\
\hline 8 & Cassia occidentalis(L) & Fabaceae & Ponnavarai & roots, leaves and seeds & Chrysophanol 1 \\
\hline 9 & Cocciniagrandis(L.)J.Viogt & Cucurbitaceae & Koovaikodi & leaves & Cephaoandrins \\
\hline 10 & DaturastramoniumL. & Solanaceae & Unmatta & Seed & 7-hydroxy apoatropine \\
\hline 11 & DioscoreaoppositifoliaL. & Dioscoreaceae & Chinese yam & tuber, flower, leaf & Cardiac glycoside \\
\hline 12 & Erigeron canadensisL. & Asteraceae & Horseweed & aerial parts of plant & $\beta$-Caryophyllene \\
\hline 13 & Guizotiaabyssinica(L.f.) Cass. & Asteraceae & Malaiellu & seeds & Campesterol \\
\hline 14 & JatrophamultifidaL. & Euphorbiaceae & Churakkalli & Whole plant & Fraxidin \\
\hline 15 & Madhucalongifolia(Koenig) & Sapotaceae & Iluppai & $\begin{array}{l}\text { Flower, leaves, bark \& } \\
\text { seeds }\end{array}$ & Stigmasterol \\
\hline 16 & MatricariarecutitaL. & Asteraceae & wild chamomile. & Flowers & Chamazulene \\
\hline 17 & Pergulariadaemia(Forsk) Chiv & Asclepiadaceae & Veliparuthi & aerial parts & Hentriacontane \\
\hline 18 & RicinuscommunisL. & Euphorbiaceae & Castor & Seed & $\mathrm{N}$-demethylricinine \\
\hline 19 & Solanumnigrum $L$ & Solanaceae & manatthakkali & whole plant & gallic acid \\
\hline 20 & Zizphusjujuba(L.) & Rhamnaceae & Ber & Fruit & Stigmasterol \\
\hline
\end{tabular}


Table 5. List of medicinal plants used by Irula tribal for the treatment of kidney disorders.

\begin{tabular}{|c|c|c|c|c|c|}
\hline S.No & Botanical Name & Family & Common Name & $\begin{array}{l}\text { Parts } \\
\text { used }\end{array}$ & Chemical constitution \\
\hline 1 & Abutilon indicum Linn. & Malvaceae & Thuthi & Leaf & $\begin{array}{l}\text { Ethylacetate, Chloroform, Methanolic, Aphrodisiac, } \\
\text { Laxative, Mucilage }\end{array}$ \\
\hline 2 & Aervalanata L. & Amarandhaceae & Kanpulai & $\begin{array}{l}\text { Root, } \\
\text { loaf }\end{array}$ & $\begin{array}{l}\beta \text {-Sitosterol, } \alpha \text {-amyrin,betulin, } \\
\text { Sitosterylpalmitate, Hentriacontane, } \\
\text { Rhamnogalactoside }\end{array}$ \\
\hline 3 & Abelmoschusesculandus L. & Malvaceae & Vendai & Fruit & Saponins, Glycosides, linoleic, linolenic ,oleic acid, squalene \\
\hline 4 & Amarandhuscaudatus L. & Amarandhaceae & Cirukeerai & Root. & $\begin{array}{l}\beta \text {-carotene.Triterpenoids, Saponins, Glycosides, linoleic, } \\
\text { linolenic ,oleic acid, squalene }\end{array}$ \\
\hline 5 & Boerhaaviadiffusa L. & Nyctaginaceae & Mukkurttaik koti & Root & phlobaphenes and ursolic acid \\
\hline 6 & $\begin{array}{l}\text { Bryophyllumpinnatum(La } \\
\text { m.)oken }\end{array}$ & Crassulaceae & Malaikali & Leaves & $\begin{array}{l}\beta \text {-D-glucopyranoside, nundecanyl, flavanoids ,flavones, } \\
\text { falvans, flavanones, isoflavonoids, chalcones, }\end{array}$ \\
\hline 7 & Coleus aromaticusBenth & Lamiaceae & Karpuravalli & Leaves & $\begin{array}{l}\text { Oleanolic acid, 2,3- dihydroxyoleanolic acid, Crategolic acid, } \\
\text { Ursolic acid, Pomolic acid, ssEuscaphic acid, }\end{array}$ \\
\hline 8 & Celosia argentia L. & Verbenaceae & Kozhikontai & $\begin{array}{l}\text { Seed, } \\
\text { root }\end{array}$ & $\begin{array}{l}\text {,6-methoxygenkwanin,quercetin, Chrysoeriol, Luteolin, } \\
\text { Apigenin, Flavanoneeriodyctol, Flavanol }\end{array}$ \\
\hline 9 & Cissampelospareira L. & Menispermaceae & Ponmusutai. & $\begin{array}{l}\text { Leaf, } \\
\text { root }\end{array}$ & $\begin{array}{l}\text { 7, 12-dimethylbenz(a)anthracene (DMBA),polycyclic } \\
\text { aromatic hydrocarbon (PAH), peroxides, }\end{array}$ \\
\hline 10 & Clerodendrumserratum L. & Lamiaceae & Thalunarai & Leaf & calcium, magnesium ,uric acid, carbohydrates \\
\hline 11 & Dolichosbiflorus L. & Fabaceae & Kollu & Root & $\begin{array}{l}\text { petroleum ether, Alcohol, Calcium chloride dehydrate, } \\
\text { Sodium oxalate, Disodium hydrogen phosphate }\end{array}$ \\
\hline 12 & Gompherna serrate L. & Amarandhaceae & Arasan con todo & $\begin{array}{l}\text { Whole } \\
\text { plant }\end{array}$ & $\begin{array}{l}\text { sulphur, chlorine, potassium, calcium, chromium , } \\
\text { manganese, cobalt, Nickel, copper, Zinc }\end{array}$ \\
\hline 13 & Hemidesmusindicus L. & Asclepiadaceae & Nanari & Root & $\begin{array}{l}\text { 4-hydroxy-3-methoxy-cinnamic acid, 4-hydroxybenzoic } \\
\text { acid,p-hydroxycinnamic }\end{array}$ \\
\hline 14 & Lagenariasiceraria (L.) & Cucurbitaceae & Surakkai & Fruit & $\begin{array}{l}\alpha-\quad \text { and } \beta \text {-amyrins, calcium albumin and alanin } \\
\text { transaminase, } \beta \text {-D-glucopyranoside }\end{array}$ \\
\hline 15 & Moringa oleifera L. & Moringaceae & Murungai & Root & alkaloids, moriginine, bacteriocide, spirochin, vitamins \\
\hline 16 & MomordicacharantiaL. & Cucurbitaceae & Pakarkai & Leaves & Alkaloid, glycosides, reducing sugar, saponin \\
\hline 17 & Phyllandhusniruri (L.) & Phyllandhaceae & Keezhanelli & Root & Alkaloid, glycosides, reducing sugar, saponin phosphatase \\
\hline 18 & Scopariadulcis (L.) & Scrophuraliaceae & Sarkaraivempu & $\begin{array}{l}\text { Root, } \\
\text { shoot }\end{array}$ & Calcium chloride, sodium oxalate, calcium chloride. \\
\hline 19 & 'I'ribulusterrestis L. & Zvgophvllaceae & Nerunii & $\begin{array}{l}\text { Whole } \\
\text { plant }\end{array}$ & $\begin{array}{l}\text { Peroxide,malondialdehyde, ethanalic,protein,carboxinyl,cat } \\
\text { alase glutathione, dithiobis, nitrobenzoic acid }\end{array}$ \\
\hline
\end{tabular}




\section{CONCLUSION}

In the present investigation, a total of 85 species of medicinal plants distributed among 80 genera belonging to 48 families were identified at Konbanur village, Anaikatti hills, the Western Ghats, Coimbatore district. In this survey Amarandaceae, Asteraceae and Solanaceae family species served as a food and Asclepiadaceae, Combretaceae, Rhamnaceae and Liliaceae, Euphorbiaceae and etc., families are utilized for various ailments. It is clearly indicates that there is wide usage of local flora by the Irulars community in study area.

This rural area is an important source of traditional medicines. More information may be explored from the peoples residing in the remote villages in this district. The traditional healers are the main source of knowledge on medicinal plants. This knowledge has been transmitted orally from generation to generation; however it seems that it is vanishing from the modern society since younger people are not interested to carry on this tradition. It is also observed that some traditional plants in that area are fast eroding. The conservation efforts are needed by plantation and protection of these plants with maximum participation of local people.

\section{REFERENCES}

Ayyanar, M. and S. Ignacimuthu, (2005). Medicinal plants used by the tribals of Tirunelveli hills, Tamil Nadu to treat poisonous bites and skin diseases. Indian J. Tradit. Know. 4: 229-236.

Census of India, 1991 and 2001.

Department of Tribal Welfare of Tamil Nadu, Statistic table, July 2006.

Gamble, J.S. (1936). Flora or the Presidency of Madras. Vol 1-3. Allard Co.London (Reprinted1956 Botanical Survey of India), Calcutta.

Ignacimuthu, S., M. Ayyanar and K. Sankarasivaraman, (2006) Ethnobotanical investigations among tribes in Madurai district of Tamil Nadu, India. J. Ethnobiol. Ethnomed. 2: 25.
Jain, S.K. (1989). Methods and approaches in Ethnobotany. Society of ethnobotany. CDRI, Lucknow, India.

Jain, S.K. and S. Shrivastava, (2001). Indian ethnobotanical literature in last two decades: A graphic review and further direction. Ethnobot. 13(1): 1-8.

Kala, C.P. (2005). Indigenous uses, Population density and Conservation of threatened medicinal plants in protected areas of Indian Himalayas. Conservat. Biol. 19(2): 368-378.

Martin, G.J. (1995). Ethnobotany: methods and manual Chapman and Hall, London.

Mathew, K.M. (1983). The Flora of the Tamilnadu Carnatic Series Vol 1-3. Ranipet Herbarium. St. Joseph's College, Trichy, p. 17-34.

Pushpangadan, P. and C.K. Atal, (1984). Ethnomedico-botanical investigations in Kerala I. Some primitive tribal of Western Ghats and their herbal medicine. J. Ethnopharmacol. 11(1): 5977.

Ragupathy, S. and S.C. Newmaster, (2009). Valorizing the 'Irulas' traditional knowledge of medicinal plants in the Kodiakkarai Reserve Forest, India. J. Ethnobiol. Ethnomed. 5: 1-13.

Sandhya, B., S. Thomas, W. Isabel and R. Shenbagarathai, (2006). Ethnomedicinal plants used by the valaiyan community of Piranmalai hills (Reserved forest), Tamil Nadu, India - A pilot study. Afr. J. Tradit. Complement. Alternat. Med. 3(1): 101-114.

Sasi, R., A. Rajendran and M. Maharajan, (2011). Wild edible plant Diversity of Kotagiri Hills - a Part of Nilgiri Biosphere Reserve, Southern India. J. Res. Biol. 2: 80-87.

Schultes, R.E. (1960). Tapering our heritage of ethnobotanical lore. Econ. Bot. 14: 157-262.

Schultes, R.E. (1962). The role of ethnobotanists in search for new medicinal plants. Llyodia 25: 257 -266 . 